\title{
Politique
}

\section{Le Québec face aux biotechnologies}

\section{Alberto Cambrosio, Charles H. Davis et Peter Keating}

Numéro 8, automne 1985

Innovations et politiques technologiques

URI : https://id.erudit.org/iderudit/040498ar

DOI : https://doi.org/10.7202/040498ar

Aller au sommaire du numéro

Éditeur(s)

Société québécoise de science politique

ISSN

0711-608X (imprimé)

1918-6584 (numérique)

Découvrir la revue

Citer cet article

Cambrosio, A., Davis, C. H. \& Keating, P. (1985). Le Québec face aux

biotechnologies. Politique, (8), 77-101. https://doi.org/10.7202/040498ar d'utilisation que vous pouvez consulter en ligne.

https://apropos.erudit.org/fr/usagers/politique-dutilisation/ 


\title{
Le Québec face aux biotechnologies
}

\author{
Alberto Cambrosio*, Charles H. Davis** et Peter Keating***
}

\section{Introduction}

Système technologique encore jeune, entouré de zones d'incertitude, les biotechnologies, dont les promesses, pour l'instant, dépassent toujours les réalisations, constituent un domaine aux contours flous, recoupant transversalement plusieurs secteurs économiques, s'exprimant par le biais de réalités institutionnelles aussi complexes que variées, et s'inscrivant d'emblée dans une dimension internationale. Cet ensemble de technologie fait depuis à peine dix ans l'objet de politiques de développement dans plusieurs pays. Préoccupation gouvernementale plus récente au Québec, l'élaboration et la mise en œuvre d'une politique biotechnologique posent des défis majeurs au pouvoir public à plusieurs égards. Le présent article dresse un bilan de l'action gouvernementale au Québec dans le domaine des biotechnologies, en faisant ressortir son orientation de même que les contraintes et les limites qui la

* Département de sociologie, Université de Montréal.

** Conseil de la Science et de la Technologie du Québec. Les opinions exprimées ici n'engagent que l'auteur.

*** Institut d'histoire et de sociopolitique des sciences, Université de Montréal. Une partie des recherches pour cet article a été rendue possible par une subvention des Fonds FCAC (85-AR-0262) ainsi que par une bourse postdoctorale de la Fondation du Prêt d'Honneur attribuée à $A$. Cambrosio. 
caractérisent. En particulier, nous allons montrer que des éléments majeurs de la dynamique du champ biotechnologique québécois échappent au contrôle du gouvernement provincial; par conséquent, le chemin emprunté par l'action gouvernementale s'écarte dans les faits du modèle de développement industriel implicitement préconisé par le programme d'intervention rendu public par le gouvernement.

On ne saurait cependant pas discuter de la situation québécoise sans avoir à l'esprit les voies que l'innovation biotechnologique a su emprunter dans d'autres pays. Avant de procéder à l'analyse de la politique biotechnologique du Québec, il apparaît donc opportun de clarifier, en quelques paragraphes, des questions «techniques» qui prêtent à confusion, notamment celles ayant trait au statut épistémologique et disciplinaire des biotechnologies, et de présenter un bref aperçu du contexte institutionnel qui a servi de cadre au développement des biotechnologies dans les principaux pays innovateurs.

\section{L'innovation en biotechnologies: une vue d'ensemble}

Malgré l'utilisation sporadique qui a pu être faite au début du siècle du terme «biotechnologies» (Dixon 1985), la diffusion de ce néologisme date de la deuxième moitié des années 1970. Elle est donc contemporaine de la mise au point dans des laboratoires de recherche biomédicale d'un ensemble de techniques «révolutionnaires» (le génie génétique, les fusions cellulaires) permettant d'effectuer des recombinaisons génétiques et de produire des anticorps monoclonaux (pour un survol de ces techniques voir Prentis 1984). Deux questions se posent d'emblée. Sommes-nous en présence de recherche appliquée ou de recherche fondamentale? Les biotechnologies se réduisent-elles à ces nouvelles techniques?

La nature des liens entre la science et la technologie a fait l'objet de nombreux débats. Dans un article posthume, Price 
(1984) a proposé de considérer un troisième domaine se situant en quelque sorte entre la science et la technologie et regroupant ce qu'il appelle des «instrumentalities», c'est-à-dire les techniques expérimentales et les appareils scientifiques. Des percées dans ce domaine auraient des répercussions aussi bien sur le versant plus proprement scientifique que sur le versant des innovations technologiques d'intérêt industriel, et constitueraient donc un des moteurs de l'évolution de ces deux ensembles tout en établissant un pont entre eux. Les nouvelles biotechnologies semblent s'inscrire assez bien dans ce schéma, par ailleurs criticable (Freeman 1982, 16). D'un côté, elles se sont imposées dans la recherche fondamentale: comme le note Panem (1984, 94), le scientifique qui, tout en travaillant dans les domaines concernés par les nouvelles techniques, voudrait en faire l'économie, serait vite pénalisé au niveau des résultats mais aussi (et corrélativement) au niveau des publications et des subventions. De l'autre, ces mêmes techniques de base peuvent être utilisées dans la mise au point de nouveaux produits ou de nouveaux procédés de production dans un ensemble de secteurs industriels. On peut donc remarquer, et ceci est important pour la suite de notre étude, que les biotechnologies présentent un intérêt certain, mais distinct, pour les milieux scientifiques universitaires et pour les mileux industriels.

Les biotechnologies ne se réduisent pas à ce que Goma et Monsan (1983) appellent les techniques "d'amont», c'est-à-dire le génie génétique et les fusions cellulaires. A quelques exceptions près, pour que les applications de ces dernières atteignent le marché, il faut avoir recours à un ensemble de techniques «d'aval» que l'on peut regrouper sous le terme «bioingénierie» («bioprocess technology») et qui incluent, notamment, les techniques de fermentation. Leur utilisation consciente, par exemple dans des industries comme les brasseries, remonte au siècle passé (Penasse 1981); par rapport aux techniques "d'amont», il s'agit donc de techniques «traditionnelles» qui n'ont été que rétrospectivement regroupées 
sous le terme «biotechnologies». Il faut cependant s'empresser d'ajouter que, d'une part, le génie des procédés biologiques a connu, au cours des dernières années, de profondes transformations, notamment grâce à l'utilisation de la micro-électronique (OTA 1984, 44-57) et que, de l'autre, l'expérience accumulée dans ce domaine par des industries traditionnelles s'est révélée très utile pour le développement des procédés plus récents: le cas du Japon est, à cet égard, très éloquent (voir plus loin).

Le besoin de disposer de statistiques comparatives, mais aussi de cerner un domaine qui semble échapper aux catégories traditionnelles de la politique scientifique et technologique, a poussé de nombreux comités et organismes officiels à proposer des définitions plus ou moins englobantes du terme «biotechnologie». Souvent, celles-ci n'ont fait qu'ajouter à la confusion (pour des exemples de définitions, voir Bull et al 1982, 67 et U.S. Department of Commerce 1984, 212-213). D'un point de vue commercial, la distinction entre techniques «d'amont» et techniques «d'aval» nous semble plus décisive que celle entre «anciennes» et «nouvelles» biotechnologies. Le génie génétique et les fusions cellulaires, en tant que techniques de laboratoires, peuvent être appliquées à la mise au point d'innovations s'adressant à un ensemble varié de secteurs économiques. Des faiblesses dans le domaine des bioingénieries et pouvant aussi bien concerner la disponibilité de maind'œuvre spécialisée que celle d'équipements sophistiqués et du savoir-faire qui s'y rattache, constituent toutefois dans bien des cas des obstacles sérieux à la mise en valeur industrielle des percées effectuées en laboratoire.

Les biotechnologies, nous venons de le voir, ne renvoient pas à un secteur industriel ou commercial particulier mais constituent plutôt un ensemble de techniques potentiellement applicables à plusieurs secteurs. Parmi ceux-ci, le secteur biomédical (et, à l'intérieur de celui-ci, le domaine des produits diagnostics) a sans doute été le premier à avoir été touché par les techniques mises 
au point au cours des années 1970. Les autres secteurs où les biotechnologies devraient connaître des applications, soit en remplaçant les procédés traditionnels de fabrication, soit en réalisant de nouveaux produits, incluent l'agriculture (animaux et végétaux), l'industrie chimique (spécialités chimiques, additifs alimentaires ainsi que produits de grande consommation), l'environnement, la foresterie, la transformation de la biomasse et la bioélectronique (OTA 1984, 119-257). Les premières réalisations biotechnologiques ont désormais atteint le stade du marché (Business Week 1984; Rowan 1985): si certaines applications peuvent paraître encore hypothétiques (par exemple la bioélectronique), on ne saurait plus longtemps douter de la réalité commerciale des nouvelles technologies.

D'un point de vue géographique, les trois principaux pôles de développement des biotechnologies sont les États-Unis, le Japon et l'Europe; dans ces trois régions, on retrouve une dynamique bioindustrielle différente. Horwitch (1984) propose de distinguer entre trois «modes» d'innovation biotechnologique. Le mode 1 renvoie aux entreprises de haute technologie et de petite taille, le mode 2 aux grandes entreprises "possédant plusieurs divisions, de multiples produits et divers marchés", et le mode 3 aux entreprises «regroupant plusieurs entités et faisant intervenir, en règle générale, le secteur public et le secteur privé dans le cadre de vastes programmes correspondant à un objectif précis». Les entreprises du mode 1 et du mode 2 correspondent, respectivement, aux «nouvelles firmes de biotechnologie» («entrepreneurial ventures started to commercialize innovations in biotechnology») et aux «compagnies établies» («process oriented, multiproduct companies in traditional industrial sectors») dont fait état un rapport de l'Office of Technology Assessment américain (OTA 1984, 61-113). alors que l'existence du mode 3 s'explique principalement par la volonté de certains gouvernements d'intervenir directement dans le développement des biotechnologies industrielles. 
Les trois modes décrits par Horwitch constituent en quelque sorte des «types idéaux» dont les attributs, dans la réalité, tendraient à s'estomper dans la mesure où, comme nous le verrons plus loin, des liens de plus en plus étroits se nouent entre les firmes des modes 1 et 2 . Il s'agit pourtant de catégories fort utiles, nous permettant de faire ressortir des différences nationales. Ainsi, les entreprises du mode 1 se retrouvent surtout aux États-Unis, où elles ont été incontestablement à l'origine de la vague d'innovations biotechnologiques. Les entreprises du mode 2 de retrouvent aussi bien aux États-Unis qu'en Europe et au Japon. Aux États-Unis, cependant, pour ce qui et de l'innovation, elles ont d'abord été à la remorque des entreprises de type 1 et ce n'est que plus récemment (surtout à partir du début des années 1980) qu'elles ont augmenté leurs activités internes de R-D biotechnologique. $\mathrm{Au}$ Japon, pays qui possède une longue tradition industrielle dans le domaine des fermentations, ce sont par contre les entreprises du type 2 qui ont joué le rôle de moteurs de l'innovation biotechnologique. Quant au mode 3, il est surtout présent au Japon et en Europe, avec l'établissement par les différents gouvernements d'une politique biotechnologique nationale. Une trace assez éloquente de ces efforts de programmation se retrouve dans les nombreux rapports commandités par les gouvernements, notamment en France, en Angleterre et en Allemagne (pour une liste de ces rapports voir Sasson 1983, 256-257; pour apprécier la façon dont les politiques nationales s'articulent différemment sur les institutions de type 1, 2, et 3 voir l'étude de cas de Jasanoff 1985 et celui de Rothman et Towalski 1984).

Étant donné l'attrait exercé au Québec par le «modèle américain", il nous semble opportun d'y consacrer quelques lignes additionnelles. La création, à partir de 1976, d'un très grand nombre d'entreprises du mode 1 a été rendue possible par deux facteurs: d'une part, la disponibilité de capital de risque ( «venture capital») et, de l'autre, la présence de scientifiques-entrepreneurs, 
c'est-à-dire d'universitaires prêts à se lancer dans des aventures commerciales. Ces deux facteurs ne suffisent toutefois pas à rendre compte de l'essor des nouvelles firmes de biotechnologie. Le capital de risque ne parvenant pas à lui seul à couvrir les coûts croissants de la R-D et de la commercialisation, on a de plus assisté à la mise en ouvre d'un ensemble de liens complexes (licences, contrats de développement de produits spécifiques, et, par la suite, prises de participation et "joints ventures») entre les entreprises du mode 1 et les entreprises du mode 2. Il serait donc faux de concevoir les nouvelles compagnies de biotechnologie comme une sorte d'alternative aux grandes compagnies traditionnelles: en réalité, il y a complémentarité et, comme le note un rapport de l'OTA (OTA 1984, 103), le succès de l'innovation biotechnologique aux États-Unis découle précisément de la présence simultanée de ces deux types d'entreprises et de la dynamique qui les unit.

Les nouvelles compagnies ont d'abord fonctionné comme des «usines de R-D», la production et la distribution des produits découlant de leurs activités étant assurées par des entreprises multinationales. Par la suite, les plus importantes parmi les nouvelles compagnies ont changé de stratégie et visent désormais l'intégration verticale de leurs opérations. Pour ce qui est du personnel, ceci se traduit par une diminution parfois drastique du nombre de scientifiques au profit du personnel administratif ainsi que par le remplacement, à la tête des compagnies, des scientifiques-entrepreneurs par des cadres professionnels. Afin de couvrir les coûts de création des unités de production, les nouvelles compagnies ont d'abord eu recours à des offres publiques d'actions et ensuite, le marché boursier se détériorant, au partenariat en $R-D$ ( $R \& D$ limited partnership»). Cette dernière source de financement ne s'est pas révélée aussi efficace que prévu, ce que d'aucuns ont interprété comme un symptôme du désenchantement des investisseurs envers des entreprises dont les produits tardent à atteindre le marché et qui sont désormais menacées par l'attitude de plus 
en plus aggressive des grandes compagnies (The Globe and Mail 1984).

Ces dernières, en effet, ont considérablement augmenté leur propre effort de R-D biotechnologique et, comme le montre la poursuite que Hydbritech a intenté contre la multinationale Abbott sur la base de la loi anti-trust (Newswatch 1985a), elles ne sont pas disposées à abandonner le marché aux nouvelles compagnies. Le phénomène de disparition d'un grand nombre d'entreprises du type 1, que l'on prédit depuis longtemps, tarde toutefois à se produire, l'épreuve du marché ne jouant pas encore pleinement son rôle: on s'accorde cependant pour reconnaître qu'il est imminent. Récemment, d'ailleurs, les représentants de l'Industrial Biotechnology Association, qui regroupe les plus importantes parmi les nouvelles compagnies aux États-Unis (par opposition à l'Association of Biotechnological Companies, qui réunit les nouvelles entreprises de moindre envergure) ont affirmé qu'après une courte période de crise, "les bons vieux jours étaient de retour»: il est toutefois révélateur que cette remarque ne s'applique pas, comme jadis, au domaine dans son ensemble mais, surtout, aux cinq compagnies qui, à elles seules, ont généré, en 1984, $75 \%$ des revenus (Newswatch 1985).

\section{La politique québécoise en biotechnologies: un bref historique}

En 1979, Pierre Sormany, vulgarisateur scientifique devenu, par la suite, conseiller scientifique du ministre de la Science et. de la Technologie Gilbert Paquette, lance un premier «cri d'alarme» au Québec, en exprimant la crainte que le pays rate l'occasion de bâtir une structure bioindustrielle (Sormany 1979). Dans son document, intitulé de façon imagée Les micro-esclaves: vers une industrie canadienne, l'auteur propose l'adoption d'un ensemble impressionnant et fort hétérogène de mesures destinées à favoriser l'éclosion des applications biotechnologiques au Canada. 
En juin 1980, le gouvernemental fédéral établit un groupe de travail présidé par Maurice Brossard, alors directeur du développement à l'Institut Armand-Frappier. Le mandat de ce comité est d'«identifier les domaines de R-D qui présentent le plus d'avantages pour le Canada et d'étudier les moyens possibles d'encourager la R-D nécessaire et de s'assurer qu'elle soit utilisée dans le but de répondre aux besoins socio-économiques du pays». Le rapport, déposé en février 1981 (Rapport du groupe de travail sur la biotechnologie 1981) recommande l'établissement d'un centre national de recherche en biotechnologie, l'élaboration d'un plan décennal national dans lequel le gouvernement fédéral jouerait un rôle de coordonnateur, et des engagements financiers importants (en septembre 1981, Brossard parlait d'un investissement d'un demi-milliard de dollars; voir Sauvé 1981).

Sans attendre l'élaboration définitive d'un plan national, plusieurs provinces se lancent en biotechnologies. Particulièrement remarquable est la création en 1982 de la compagnie Allelix dans la région de Toronto par le gouvernement de l'Ontario, en association avec John Labatt Ltd. et le Canada Development Corporation; les responsables de ce centre de R-D lancent une campagne internationale de recrutement de leur personnel scientifique (Moriarity 1983a).

Au Québec, le Secrétariat au développement scientifique effectue à partir de l'hiver 1981 une étude sur l'état de la recherche en biotechnologies dans la province. Les travaux du Secrétariat débouchent sur une version préliminaire d'un programme quinquennal d'intervention qui circulera pour commentaires en décembre 1981; la version finale est rendue publique sous le nom de $\grave{A}$ l'beure des biotechnologies en octobre 1982 (SSTQ 1982). Ce programme prévoit quatre ensembles de mesures:

1) le développement des ressources humaines; recyclage des chercheurs, programme de bourses, création de postes universitaires, mise sur pied d'unités spéciales de formation; 
2) l'organisation des transferts vers l'industrie (dont on admet par ailleurs la presque inexistance) grâce notamment à l'établissement d'une société de recherche et de développement;

3) le financement de la R-D: la programme prévoit une mobilisation de $63 \mathrm{M} \$$;

4) la mise en place d'un dispositif de gestion: on prévoit la création d'un Comité ministériel ad hoc pour la coordination intra-gouvernementale, d'une mission de concertation pour l'ensemble des intervenants, et de cinq missions ad hoc composées de spécialistes.

En mai 1983 le gouvernement fédéral annonce la mise sur pied à Montréal d'un centre national de R-D en biotechnologie. Disposant d'un budget annuel de fonctionnement de $40 \mathrm{M} \$$ et devant accueillir jusqu'à 300 chercheurs et techniciens, ce centre se classerait parmi les plus grands établissements publics de R-D biotechnologique au monde. Ottawa annonce en même temps le dévoilement prochain d'une stratégie nationale en biotechnologies. Plus de deux ans après la présentation du «rapport Brossard», le gouvernement fédéral en applique ainsi les principales recommandations (Chemistry in Canada 1983).

L'année 1983 voit un renforcement du pouvoir d'intervention en biotechnologies de plusieurs organismes publics québécois. La Société générale de financement (SGF), organisme étatique d'investissement créé en 1960 et actif surtout dans les domaines de l'équipement énergétique, des produits forestiers et de la pétrochimie, reçoit à la fin de 1982 le mandat d'œuvrer également dans le domaine des biotechnologies. Elle acquiert l'Institut de Bio-endocrinologie, entreprise montréalaise fabricant des trousses diagnostique, et elle crée une filiale, Bio-Méga, une société de recherches en commandite, d'investissement et de commercialisation en biotechnologies qui détient $70 \%$ de Bio-Endo. L'autre $30 \%$ de Bio-Endo appartient à Biocel, un organisme de recherche à but non lucratif regroupant des universitaires québécois. 
Egalement en 1983 sont établis le Ministère de la Science et de la Technologie (MTS), organisme ayant des responsabilités directes en matière de politique biotechnologique, et deux organismes publics de capitaux de risque, l'Agence québécoise pour la valorisation de la recherche industrielle (AQVIR) et BIO-AGRAL. Cette dernière dispose d'un capital de $10 \mathrm{M} \$$ pour l'achat d'actions ordinaires de firmes innovatrices œuvrant dans le domaine de l'agro-alimentaire. De plus, le Centre de recherche industrielle du Québec (CRIQ) se voit confier un mandat de R-D en biotechnologies.

À l'exception du programme d'actions structurantes en milieu universitaire, se proposant de financer la formation d'équipes de recherche dans les domaines prioritaires reliés au «Virage technologique», et d'un projet de mise sur pied d'un Centre québécois pour la valorisation de la biomasse, ce sera la fin d'une vague de création d'institutions publiques en biotechnologies. Le problème est maintenant de savoir comment allouer les ressources prévues, à l'intérieur du cadre politique et institutionnel ainsi établi. Les nombreux énoncés de priorités en biotechnologies émis par les nouvelles institutions et par les agences publiques sont en effet assez vagues pour laisser une grande marge de manœuvre quant aux orientations précises susceptibles d'être subventionnées.

Forces et faiblesses de la politique québécoise en biotechnologies

Un bilan des réalisations gouvernementales québécoises en matière de développement biotechnologique rendu public par le ministère de l'Enseignement supérieur, de la Science et de la Technologie en 1985 indique que depuis 1982, le Québec a consacré environ $53 \mathrm{M} \$$ à diverses mesures reliées à ce domaine (MESST 1985). De cette somme, approximativement 26,5\% a servi à soutenir la formation et la recherche universitaires et des 
interventions ad hoc, environ 19,6\% a été dépensé par les ministères sectoriels aux fins de recherches commanditées ou de recherches intra-muros, et presque $54 \%$ a été consacré au développement des activités biotechnologiques des sociétés d'état, notamment Bio-Méga (MESST 1985, 11).

Cette répartition des fonds soulève plusieurs questions quant à la stratégie québécoise en matière de développement biotechnologique. En effet, le document $\grave{A}$ l'beure des biotechnologies avait comme but d'esquisser uniquement la première phase d'une stratégie de bioindustrialisation, et à ce titre ne prévoyait pas le développement d'un rôle aussi prononcé pour le gouvernement en tant qu'intervenant bioindustriel. Dans ce qui suit nous allons comparer le modèle de développement biotechnologique implicitement préconisé par À l'heure des biotechnologies à celui qui a été adopté dans les faits par le gouvernement et nous allons identifier des facteurs qui rendent problématique la programmation gouvernementale du développement des biotechnologies au Québec.

1. Politique biotechnologique et politique industrielle. En établissant une politique qui vise à promouvoir l'innovation technologique, un gouvernement reconnaît, implicitement ou explicitement, que le changement technologique constitue un élément essentiel du développement économique à long terme et, en particulier, après la crise qui a frappé les pays occidentaux, un élément de la relance économique. L'intervention gouvernementale dans le domaine des biotechnologies, au-delà de l'intérêt plus proprement scientifique de celles-ci, n'acquiert donc tout son sens que dans le cadre d'une politique industrielle. Or, si le gouvernement du Québec, dans les documents consacrés aux biotechnologies, a maintes fois souligné que la raison d'être de son intervention était économique et qu'elle visait à susciter la bioindustrialisation, on peut difficilement par contre affirmer que l'action gouvernementale, aussi bien pour ce qui est des mécanismes décisionnels dont elle s'est servie qu'en ce qui concerne les contenus 
spécifiques qu'elle s'est donnés soit toujours articulée sur des stratégies de développement industriel.

Les mesures annoncées pour la première phase du programme d'intervention (SSTQ 1982) visent explicitement le développement de la formation et de la recherche en amont des industries et la promotion de transferts vers des industries, en grande partie hypothétiques. Ces mesures renvoient à une conception de l'innovation biotechnologique qui s'apparente au «mode I» de Horwich, mode qui privilégie les investissements en recherche fondamentale dans les universités et l'apport de capitaux de risque pour les petites firmes que essaimeront autour de celles-ci. Ainsi que nous l'avons déjà signalé, le mode I représente un phénomène organisationnel typiquement américain qui a rarement été reproduit dans d'autres pays. La stratégie industrielle du programme québécois en biotechnologies nous paraît représenter une tentative de susciter au Québec une dynamique de bioindustrialisation semblable à celle existant aux États-Unis à la fin des années 70 .

Si le développement de petites firmes biotechnologiques autour des universités québécoises demeure possible et même souhaitable, une telle structure bioindustrielle est loin de caractériser le Québec à l'heure actuelle. En plus, l'idée même d'une bioindustrialisation axée sur le mode I est en quelque sorte problématique dans la mesure où les firmes de ce genre sont de plus en plus obligées de traiter avec les firmes du mode 2 , les multinationales. La stratégie québécoise en matière des biotechnoligies telle qu'énoncée dans $\grave{A} l$ l'beure des biotechnologies ne tient pas compte de ces intervenants importants.

Dans les faits, la stratégie de bio-industrialisation adoptée par le Québec ressemble au mode 3 de Horwich. Cependant, il est important de noter que la mise sur pied de Bio-Méga ne correspond pas à l'établissement de la Société de recherche et de développement en biotechnologies préconisée par le programme gouvernemental d'intervention (SSTQ 1982, 35): plutôt que d'une 
association entre des entreprises publiques et privées, il s'agit d'une organisation entièrement reliée au secteur public. D'autre part, dans un premier temps Bio-Méga avait été conçue uniquement comme une société de financement, ne possédant donc pas ses propres structures de recherche, de développement et de production. C'est suite à une décision motivée par des facteurs conjoncturaux que la vocation de Bio-Méga a été modifiée (voir plus loin).

2. La structure économique du Québec en tant que contrainte. $S$ 'il est acquis que les interventions gouvernementales sont motivées principalement par le désir de susciter de développement de bioindustries avancées, il n'est par contre pas facile de déterminer quelles mesures gouvernementales d'incitation seraient les plus indiquées pour suppléer à la quasi-absence d'acteurs industriels capables d'adopter les innovations biotechnologiques produites dans les universités et dans les laboratoires gouvernementaux. L'adoption par le Québec d'une stratégie de bioindustrialisation axée de façon significative sur la prise en charge étatique d'un ensemble d'activités depuis la R-D jusqu'à la commercialisation implique tant le choix d'un mode d'intervention que le choix des domaines sectoriels dont l'intervention gouvernementale vise à privilégier le développement. L'analyse des avantages et des inconvénients de ces choix, rendus difficiles par l'incertitude qui entoure l'évolution technique et l'évaluation des marchés, devrait figurer explicitement dans l'élaboration de politiques biotechnologiques et bioindustrielles ultérieures.

Au Québec, parmi les secteurs industriels susceptibles d'une éventuelle mise en valeur des percées biotechnologiques, l'industrie de la santé est de loin celle qui peut le plus compter sur la présence d'activités de R-D, assurées par divers organismes publics et universitaires et par quelques firmes privées. Or, contrairement à l'Ontario, où l'industrie pharmaceutique se caractérise par l'existence de firmes locales de produits génériques (pratique rendue possible par l'article 41 de la loi canadienne des brevets), l'industrie 
pharmaceutique au Québec est dominée par les entreprises multinationales. Depuis dix ans, cette industrie subit une vague de fermetures de laboratoires de R-D: Smith, Kline \& French ferme ses laboratoires en 1976, suivi de Pharma, Syntex, Abbott, Ayerst, et Hoffman LaRoche. On considère que la législation canadienne concernant l'octroi obligatoire de licences est une des principales causes de certains de ces départs, explication bien sûr contestée par les manufacturiers de produits génériques. Sans entrer dans les détails de cette question complexe (voir Eastman 1985), on peut noter que le gouvernement du Québec, en réclamant la modification de la loi canadienne des brevets, endosse à toute fin pratique la position des grandes compagnies pharmaceutiques.

La fermeture en 1982 des laboratoires de recherche de la firme Ayerst et les efforts du gouvernement du Québec pour relocaliser les chercheurs de cette entreprise illustrent bien la façon dont des décisions prises à l'extérieur du Québec peuvent provoquer une réorientation majeure d'une politique québécoise en biotechnologies. En juin 1982, la firme Ayerst McKenna \& Harrison Ltd., filiale d'une société américaine, annonçait le déménagement prochain vers les États-Unis de l'un des plus importants laboratoires de recherche pharmaceutique au Québec. Seulement une trentaine des chercheurs étaient invités à se relocaliser aux États-Unis; 180 chercheurs et techniciens perdaient ainsi leur emploi. En plus de provoquer un vif débat sur la loi canadienne des brevets (dont les contraintes avaient été invoquées par Ayerst pour justifier son départ), cet événement suscita une série d'interventions et de démarches tant de la part des chercheurs concernés que dans les milieux pharmaceutiques et gouvernementaux québécois. À la suite de la faillite des négociations qui visaient à intégrer dans l'Institut Armand-Frappier et dans des firmes pharmaceutiques privées une partie des ex-chercheurs de Ayerst, le gouvernement décidait de rattacher ce qui restait de l'équipe Ayerst à Bio-Méga, 
qui se voyait également allouer une importante somme pour construire des laboratoires de recherche à Laval.

La stratégie décrite dans $\grave{A}$ l'beure des biotechnologies visait principalement à accroître l'offre de biotechnologies au Québec, en intervenant presque exclusivement en amont de l'activité industrielle. Cette stratégie a donc été sensiblement modifiée dans les faits lorsque la société de financement des recherches préconisée par le document gouvernemental (cette société "stimulerait par sa politique contractuelle des recherches orientées", mais ne posséderait pas «dans une première phase d'exploitation de laboratoires de recherche»; SSTQ 1982, 35) est devenue, suite à la récupération d'une partie des chercheurs d'Ayerst, un exécutant de la recherche. La tendance à l'intégration verticale s'est par ailleurs poursuivie lorsque Bio-Méga a englouti sa propre filiale Bio-Endo (désormais appelée Bio-Méga Diagnostics), dotée de structures de production et de distribution. Le sauvetage de l'équipe Ayerst coûtera environ $47 \mathrm{M} \$$ sur une période de cinq ans; par comparaison, le soutien gouvernemental prévu dans le plan quinquennal pour le développement biotechnologique au Québec (SSTQ, Tableau 1) s'élève à environ $23 \mathbf{M} \$$ (subventions aux universités exclus). D'autres aspects et conséquences de l'opération de sauvetage se prêtent à des critiques. Si l'on compare par exemple Bio-Méga à la compagnie créée par le gouvernement ontarien, Allelix, on s'aperçoit qu'alors que la première, en intégrant les chercheurs d'Ayerst, a été obligée de composer avec leurs domaines d'expertise, qui ne se situent pas nécessairement à l'intérieur des biotechnologies au sens étroit du mot, les responsables d'Allelix ont d'abord défini des objectifs et une stratégie de recherche et ont par la suite engagé le personnel qui était le mieux en mesure de contribuer à réaliser les buts que l'entreprise s'est donnée.

Les interventions du gouvernement dans le développement biotechnologique des secteurs forestier et agro-alimentaire sont moins spectaculaires et se conforment au schéma tracé par $A$ l'beure 
des biotechnologies. Ces deux secteurs nécessitent des investissements à long terme bien en amont de l'activité industrielle avant qu'une mise en valeur commerciale des biotechnologies ne puisse être envisagée. Le contrôle étranger de ces secteurs est plus faible que dans l'industrie pharmaceutique. Tandis que le secteur privé assure un effort de R-D en biotechnologies dans le cadre de l'industrie des pâtes et papiers au Québec, le développement d'applications biotechnologiques dans le domaine de l'agro-alimentaire se heurte au problème de l'absence d'entreprises dotées d'une forte capacité technique. Lors de la séance publique du Conseil de la Science et de la Technologie tenue en novembre 1984 et consacrée au développement des bioindustries, des intervenants gouvernementaux ont fait remarquer qu'en dépit de l'existence d'un dispositif public d'investissements et de capitaux de risque qui cherche activement à soutenir les initiatives privées en matière de bioindustries avancées, il n'y avait eu jusque là que peu de projets valables à appuyer (CSTQ, 1985, 20-21). Ceci semble surtout être le cas dans le secteur agro-alimentaire, où seulement quelques firmes québécoises effectuent de la R-D. On pourrait s'attendre à ce qu'un organisme comme le CRIQ permette à des petites compagnies qui ne peuvent investir beaucoup d'argent dans la R-D de profiter du changement technologique; le CRIQ se charge de la R-D, en en partageant les frais avec la compagnie qui l'a commanditée. Toutefois, comme le remarque un de ses responsables: "[1]'absence d'une industrie biotechnologique au Québec rend impossible l'obtention de contrats de recherche par le CRIQ»(Drouin 1984, 39).

La faiblesse de la structure bioindustrielle au Québec pose ainsi un défi de taille aux responsables de la politique biotechnologique. La tentation d'intervenir en créant des industries est forte; d'autre part, le gouvernement, s'il a acquis une certaine expérience, au demeurant pas toujours très probante, en tant qu'acteur industriel dans les secteurs traditionnels (acier, textiles, 
construction de navires, amiante, etc.), n'est guère plus expérimenté que le secteur privé pour ce qui est des bioindustries de pointe.

3. Les contraintes sociopolitiques. Si les particularités de la structure économique québécoise rendent problématique l'articulation d'une stratégie de développement biotechnologique sur une stratégie de bioindustrialisation, la multiplicité des acteurs dans le champ biotechnologique québécoise constitue un défi majeur pour ce qui est de l'élaboration d'une politique de l'innovation biotechnologique et de sa mise en ouvre. Le contexte du développement des biotechnologies au Québec se caractérise par la présence d'un nombre élevé d'intervenants - universitaires, universités, instituts de recherche, groupes de pression régionaux, chambres de commerce, organismes professionnels, etc. - qui cherchent à se faire reconnaître une vocation en biotechnologies. Ces intervenants réclament des ressources et agissent souvent en dehors du cadre politique tracé par le gouvernement provincial.

La concertation est d'autant plus difficile à réaliser, qu'il faut compter sur l'existence de deux paliers gouvernementaux. Les universités, qui dépendent des subventions gouvernementales pour financer leurs activités, se livrent à une concurrence entre elles pour attirer des fonds (voir par exemple Bissonnette 1984). Une gamme de groupes de pression interviennent dans le but d'influencer les décisions gouvernementales pose elle-même un problème dans la mesure où les biotechnologies recouvrent plusieurs secteurs économiques relevant de la juridiction de différents ministère. Tout à fait édifiante, à cet égard, est la tentative ratée de l'Université du Québec, appuyée par le ministre du Commerce Extérieur, sans consultation préalable avec le ministère de l'Education, de créer à Laval une école supérieure de biotechnologies (CU 1984b; Bissonnette 1983a, 1983b).

La tâche des responsables gouvernementaux est d'autant plus complexes, qu'il n'est pas toujours facile pour eux de séparer les conseils «intéressés» que leur prodiguent les différents intervenants, 
des arguments plus proprement techniques et économiques que ces derniers sont en mesure de leur fournir: en effet, les informations et l'expertise dont disposent les intervenants sont souvent supérieurs à celles que possède le pouvoir public. Ainsi la fluidité de la structure institutionnelle des biotechnologies au Québec, jointe à la complexité sur le plan conceptuel du projet de développement biotechnologique, contribuent à "politiser" le processus d'élaboration et de mise en œuvre des mesures d'intervention.

L'établissement à Montréal d'un centre national de recherches en biotechnologies du CNRC illustre bien à quel point de contexte sociopolitique affecte la mise en place de structures de R-D biotechnologique au Québec. En septembre 1982, la Chambre de commerce de Montréal amorçait une campagne visant à assurer la localisation à Montréal du Centre international de génie génétique et de biotechnologie de l'Organisation des Nations Unies pour le Développement Industriel (ONUDI). Le lobby québécois en faveur de la venue de ce centre comprenait entre autres la Chambre de comınerce de Montréal, la Communauté urbaine de Montréal, l'Ordre des ingénieurs du Québec, la Corporation professionnelle des technologues des sciences appliquées du Québec et plusieurs personnalités universitaires. La Ville de Montréal offrait gratuitement le terrain nécessaire à la construction du centre, et le gouvernement du Québec proposait à Ottawa de collaborer à son financement, en affirmant de façon un peu hâtive qu'un tel centre «s'inscrivait exactement dans les priorités scientifiques du Québec» (Provost 1982). En annonçant en février 1983, son retrait du dossier à cause de l'impossibilité d'exercer une contrôle sur les activités d'un organisme de recherche international, le gouvernement fédéral attirait en même temps l'attention sur son inaction en matière de biotechnologies et sur son peu d'empressement à donner des suites au «rapports Brossard» (Moriarity 1983b). L'annonce par Ottawa en mai 1983 de l'établissement à Montréal d'un Institut de recherche biotechnologique constitue ainsi en grande 
partie une réponse aux attentes soulevées dans des milieux montréalais par le projet de l'ONUDI. Le caractère conjoncturel de cette opération se manifeste dans le fait que les objectifs et la structure de l'institution n'avaient pas encore été précisée au moment de l'annonce de sa création. Ils ne seront connus qu'en avril 1984 (CNRC 1984).

L'existence d'un lobby provincial en faveur de la venue à Montréal d'un centre financé par le fédéral n'est pas en soi une garantie qu'un tel investissement massif s'inscrive de façon harmonieuse dans l'ensemble des mesures prises par le gouvernement du Québec et dans l'infrastructure provinciale déjà en place. Les universités et les centres provinciaux de recherche ont exprimé la crainte d'assister à un "écrémage» de leur meilleurs chercheurs au profit du centre fédéral. En outre, certains considèrent que le gouvernement fédéral aurait mieux fait d'épauler les initiatives du gouvernement du Québec, que ce soit en contribuant au sauvetage de l'équipe Ayesrt ou en consentant une aide financière aux activités biotechnologiques de la SGF, et d'injecter davantage d'argent dans les structures universitaires ou para-universitaires, en évitant ainsi des dédoublements inutiles (voir, par exemple, Beaulnes 1983). La création de l'Institut du CNRC pose donc le problème de l'agencement entre les activités de celui-ci et les activités des organismes québécois.

Montréal n'est pas la seule ville québécoise à avoir exercé des pressions pour attirer des activités biotechnologiques sur son territoire. Dans la région de Québec, le sommet socio-économique de septembre 1983 a donné lieu à une série de propositions à cet effet. La GATIQ (Groupe d'action pour l'avenir technologique et industriel de la région de Québec), qui réunit l'Univervité Laval, le CRIQ, l'INRS, la Chambre de commerce et des municipalités de la région, a favorisé la création d'un consortium de recherche dans le domaine de la biomasse et de la biotechnologie végétale. Le projet du gouvernement du Québec d'établir un Centre québécois 
de valorisation de la biomasse a fait l'objet d'une étude de faisabilité, mais le choix de l'emplacement physique du centre semble avoir posé des problèmes de concertation intra-gouvernementale.

La multiplicité des acteurs, et en particulier la présence de nombreux groupes de pression ainsi que la concurrence que se livrent les universités, doivent par ailleurs inciter à une certaine prudence dans le maniement des catégories qui sous-tendent l'ébauche d'une politique biotechnologique, le recueil de données statistiques et l'application des programmes. Comme le remarquent les membres du Comité sur la formation en biotechnologie du Conseil des Universités (CU 1984a, 385), le ministère d'État à la Science et à la Technologie avait indentifié, en 1980, 97 chercheurs dans le domaine des biotechnologies à travers le Canada. Le Secrétariat à la Science et à la Technologie du Québec, en 1981, en recensait 120 uniquement au Québec. Quant aux universités québécoises, elles déclaraient, en 1983, en employer 244. D’après le Conseil de la Science et de la Technologie du Québec (CSTQ 1984, 16), «l'apparition soudaine d'une large population de cherchers classés par leurs universités d'attache comme biotechnologistes est étonnante». A l'origine d'une telle situation se trouve, bien sûr, le fait qu'en l'absence de structures disciplinaires de légitimation et de certification, personne ne sait trop exactement ce que c'est qu'un biotechnologiste. Ce qui nous semble intéressant, à cet égard, ce n'est pas tellement de faire ressortir le fait, après tout banal, qu'en l'absence de critères institutionnels bien définis chaque intervenant aura tendance, consciemment ou inconsciemment, à interpréter de la façon qui lui est la plus favorable une catégorie dont le contenu est éminemment négociable, mais, précisément, le fait qu'un processus de négociation est à la base de la construction sociale de cette même catégorie.

Les biotechnologies ne se réduisent pas à un simple événement technique: tout un travail social de médiation et de traduction (Calon et al 1983) doit prendre place pour qu'elles assument les 
contours institutionnels et cognitifs qu'elles semblent tenir de leurs seules propriétés «intrinsèques». L'ébauche et la mise en œuvre d'une politique scientifique sont inévitablement fondées sur l'utilisation de catégories discrètes (dans notre cas: la triade biosciences-biotechnologies-bioindustries; voir SSTQ 1982, 1-5) qui, pour qu'elles soient opérationnelles, doivent renvoyer à des ensembles comparables. A cet égard, la controverse sur le nombre des biotechnologistes ne constitue qu'un exemple macroscopique, et donc facilement perceptible, des ambiguïtés qui guettent l'établissement d'une intervention dans un domaine qui non seulement est encore en émergence mais pour lequel, de l'avis du Conseil des Universités, il faut se garder, pour l'instant, de prévoir des structures d'encadrement rigides. Des négociations aussi importantes, bien que moins évidentes, peuvent se dérouler à l'intérieur des centres de recherche, notamment entre les scientifiques et l'administration (Cambrosio et Keating, 1985).

\section{Conclusion}

Dans cet article, nous nous sommes surtout employés à souligner les contraintes avec lesquelles l'intervention gouvernementales dans le domaine des biotechnologies doit composer. Un historique, même rapide, des événements marquants de cette intervention suffit pour montrer que des décisions prises à l'extérieur du Québec - par une entreprise privée (Ayerst) et par le gouvernement fédéral - y ont joué un rôle déterminant. L'action du gouvernement provincial assume ainsi des fois les contours d'une réaction face à des évènements à son contrôle.

Dans la mesure où une politique technologique se donne comme objectif d'agencer les pôles industriel, universitaire et gouvernemental, la quasi-absence d'une structure bioindustrielle au Québec constitue de toute évidence un problème majeur pour les responsables gouvernementaux. Ceci est d'autant plus vrai, 
qu'une telle situation se solde par un débalancement marqué du champ des forces à l'intérieur duquel se joue l'établissement d'une politique biotechonologique. Des groupes de pression dont les stratégies ne sont que faiblement déterminées par des considérations plus proprement industrielles peuvent assumer un rôle majeur. Ainsi, nous sommes tentés de croire que l'importance attribuée par les actions gouvernementales au développement des biotechnologies dans le secteur de la santé, alors que l'industrie pharmaceutique au Québec est dominée par les multinationales et traverse une période de crise profonde, s'explique par la puissance relative dont jouissent les représentants des facultés biomédicales dans le champ universitaire.

La faiblesse de la structure bioindustrielle soulève également des questions quant au cadre conceptuel du programme d'intervention, qui rappelle les modèles linéaires de l'innovation dont de nombreuses études ont montré les défauts, tandis que les investissements gouvernementaux majeurs du côté industriel s'écartent sensiblement des énoncés de politique biotechnologique. L'élaboration d'une stratégie bioindustrielle efficace ne saura faire l'économie d'une analyse explicite des contraintes qu'elle doit affronter ni, non plus, d'un questionnement en profondeur du statut des acteurs et de la structure des mécanismes décisionnels qui contribuent à déterminer le contenu des politiques technologiques. Agir autrement serait se condamner d'emblée à subir, plutôt qu'à maîtriser, le contexte dans lequel une telle stratégie est appellée à se développer.

\section{Bibliographie}

BEAULNES, Aurèles, 1983. «Biotechnologies: l'heure des choix (propos recueillis par Jean-Paul Chausseau ", Science \& Technologie 2(3), 22-26.

BISSONNETTE, Lise 1984. "Un malheureux épisode», Le Devoir, 18 juillet.

— 1983a. "Le racket des diplômés", Le Devoir, 2 juillet. 
1983b. "Les chats de M. Landry", Le Devoir, 13 juillet.

BULL, Alan T. et al, 1982. Biotechnology. International Trends and Perspectives. Paris: OCDE. Business Week, 1984. "Biotech Comes of Age", 23 janvier, 84-94.

CNRC, 1984. Institut de recherche en biotechnologie: plan stratégique préliminaire. Montréal: Conseil national de recherches du Canada.

CSTQ, 1985. Le développement industriel des biotechnologies au Québec. Québec: Conseil de la science et de la technologie.

1984. Le développement des bioindustries au Québec, Document de consultation sur les biotechnologies pour la 3e séance publique du Conseil. Québec: Conseil de la science et de la technologie.

$\mathrm{CU}, 1984 \mathrm{a}$. Avis du Conseil des Universités au ministre de l'Education sur la formation en biotechnologie. Sainte-Foy: Conseil des Universités.

, 1984b. Avis au ministre de l'Education sur un projet d'école supérieure des sciences de la santé et des biotechnologies de l'Université du Québec. Sainte-Foy: Conseil des Universités.

CALLON, Michel, et al, 1983. «From translation to problematic network: an introduction to co-world analysis", Social Science Information 22, 191-235.

CAMBROSIO, Alberto, et Peter KEATING, 1985. "Studying a biotechnology research centre. A note on local socio-political issues", Social Studies of Science 15, à paraître.

Chemistry in Canada, 1983. "Canada's technology policy", été, 57-58.

DIXON, Bernard, 1985. "Putting the "bio" in biotech", Neu' Scientist, 31 janvier, 38.

DROUIN, Gilles, 1984. «Les promesses de l'or vert», Québec Science 23 (2), 36-41.

EASTMAN, H.C. et al, 1985. Le rapport de la Commission d'enquête sur l'industrie pharmaceutique. Ottawa: Ministère des Approvisionnements et Services Canada.

FREEMAN, Christopher, 1982. The Economics of Industrial Innovation. Cambridge, Mass: MIT Press.

The Globe and Mail, 1984. "Biotech bloom may be fading", 15 juin.

GOMA, Gérard et Pierre MONSAN, 1983. «Les bioindustries ont-elles un avenir?", La Recherche 14, 1149-1156.

HORWICH, Mel, 1984. "La nouvelle complexité dans l'innovation technologique et la concurrence multinationale: le cas de la naissance d'une industrie bio-technique multinationale", dans Les multinationales en mutation, A. COTTA et M. GHERMAN (éds.). Paris: PUF-IRM, 108-131.

JASANOFF, Sheila, 1985. "Technological innovation in a corporatist state: the case of biotechnology in the Federal Republic of Germany", Research Policy 14, 23-38.

MESST, 1985. Les mesures scientifiques et technologiques du Virage technologique. Des engagements aux réalisations, 1982-1985. Québec: Ministère de l'Enseignement supérieur, de la Science et de la Technologie.

MORIARITY, Andrew, 1983a. "Allelix Inc. breaks new ground in biotechnology", Canadian Research 16 (7), 17-24.

, 1983b. «Biotechnology case study: Ottawa's lack of industrial policy fails high technology future", Canadian Research 16 (1), 65-66.

Newswatch, 1985a. "Hybritech sues Abbott on antitrust violations in diagnostic market ". McGrau'Hill's Biotechnology Newswatch 5 (3), 4 février, 1-2.

1985b. "Financial analysts give biofirms top marks for bottom-line progress ", McGraw-Hill's Biotechnology Newswatch 5(4), 18 février, 1-2.

OTA, 1984. Commercial Biotechnology: an International Analysis. Washington, D.C. Office of Technology Assessment, Congress of the United States. 
PANEM, Sandra, 1984. The Interferon Crusade. Washington, D.C. : The Brookings Institution.

PENASSE, L., 1981. "Réflexions sur le développement des biotechnologies", Annales des mines 187, 77-84.

PRENTIS, Steve, 1984. Biotechnology. A New Industrial Revolution. New York: George Braziller, Inc.

PRICE, Derek de Solla, 1984. "The science/technology relationship, the craft of experimental science, and policy for the improvement of high technology innovation ", Research Policy 13, 3-20.

PROVOST, Gilles, 1982. "Québec prêt à collaborer au futur centre de recherche» Le Devoir, 1 octobre.

Rapport du groupe de travail sur la biotechnologie, 1981. Biotechnologie: un plan de développement pour le Canada. Ottawa: Groupe de travail sur la biotechnologie présenté au ministre d'Etat chargé des sciences et de la technologie.

ROTHMAN, Harry, et Zbigniew TOW ALSKI, 1984. "British Biotechnology Policy", dans UK Science PolicyMaurice Goldsmith (éd.) London et New York: Longman, 3-56.

ROWAN, Geoffrey, 1985. "Biotech's first product wave rolls toward the marketplace", The Boston Herald, 10 mars.

SSTQ, 1982. A l'heure des biotechnologies. Programme d'intervention pour le développement de la recherche en biotechnologies au Québec. Phase 1:1982-1987. Québec: Secrétariat à la science et à la technologie.

SASSON, Albert, 1983. Les biotechnologies. Défis et promesses. Paris: UNESCO.

SAUVÉ, Matthieu, 1981. "La biotechnologie: des retards que le Canada se doit de rattraper", Le Devoir, 26 septembre.

SORMANY, Pierre, 1979. Les micro-esclaves: vers une bio-industrie canadienne. Montréal: Institut de Recherches politiques.

U.S. Department of Commerce, 1984. High Technology Industries. Profiles and Outlooks. Biotechnology. Washington, D.C. 